\title{
Original
}

\section{Transport of Coarse Particles in a Fine Suspension Medium}

\author{
by \\ S.N. Bhattacharya ${ }^{\dagger}$, C. Chin $^{\dagger}$, L. Pullum ${ }^{\dagger}$ and J.O'Donnell ${ }^{\dagger}$
}

\begin{abstract}
The rheology of suspensions of coarse particles in non-Newtonian fluids has not been the subject of much investigation. There are distinct advantages in using non-Newtonian carrier fluids in the transportation of solids, for example in coal slurry transport. The objective of this work is to investigate the flow behaviour exhibited by a concentrated mixture of coarse particles in a medium consisting of suspensions of fine particles. Steady state viscosity measurements of suspensions of mono-sized coal, ballotini and styreneacrilonitrile particles suspended in a non-Newtonian bentonite clay carrier fluid have been made using a Contraves Rheomat 115 rheometer. Measurements were carried out with various particle concentrations and shear rates ranging from $2.22 \mathrm{~s}^{-1}$ to $345 \mathrm{~s}^{-1}$. It was found that variation of viscosity due to coarse particle size did not follow any trend. It is thougth that this behaviour in due to the interaction between the clay flocs and the coarse particles. Modelling work assuming interacting coarse particles and flocs coexisting as in a bimodal mixture was shown to simulate this behaviour.
\end{abstract}

Key Words : Rheology, Suspension, Viscosity, Particle Size Distribution

\section{INTRODUCTION}

The transport of a slurry of coarse particles may be complicated if the particles are of settling nature. A slurry may be stabilised if a non-Newtonian carrier fluid having yield stress is used to keep the coarse particles dispersed. In this case, if the yield stress of the fluid can counter the settling force acting on the particle, the particle will be prevented from settling. It is widely known that concentrated suspensions of fine particles $(\langle 10 \mu \mathrm{m})$ often exhibit yield stress. The effect of particle size has been the suaject of many investigations ${ }^{1,2,3}$. Small particles interact with each other wit hin the suspending medium forming flocs, agglomerates etc. Particle-particle interaction gives rise to the formation of yield stress. As the particle size increases, the interparticle force decreases and so does the yield stress. In industry it is not uncommon to have a slurry consisting of such particle size distribution that the interacting smaller size particles will be 'reactive', forming a floc-like structure and developing yield stress while the large parsicles will remain 'nonreactive' within this flo-

\footnotetext{
Received December 10, 1993

$†$ Rheology and Material Processing Center Chemical and Metallurgical Engineering Royal Melbourne Institute of Technology Melbourne, Victoria, Australia
} 
ccular system. Thus in such a slurry the larger or the coarser particles could be held in suspension by a non-Newtonian carrier fluid formed by the interactions of the fines present in the slurry. Delineation between these two types of behaviour appears to be dependent upon the fluid medium and various physico-chemical properties of the particles dispersed in this medium. The degree of non-Newtonian behaviour of the carrier fluid is dependent upon concentration, shear, particle-particle interaction, particle size and sphericity.

Recent work ${ }^{4,5,6}$ on the transport of coarse coal and mineral slurries using a non-Newtonian carrier indicates that the viscosity of the slurry is affected by the size of the coarse particles. For coalclay suspensions Renehan et $\mathrm{al}^{4}$ observed that coarse coal particles less than $600 \mu \mathrm{m}$ influenced the flow behaviour and that a minimum viscosity occurred when the particles were in the range of 100 to $150 \mu \mathrm{m}$. Further work by Chim et al $^{5}$ using ballotiniclay suspensions also confirmed this anomalous behaviour where viscosity decreased with increase in particle size $(0-90 \mu \mathrm{m})$, then increased with increase in particle size $(90-180 \mu \mathrm{m})$ and finally again decreased with further increase in particle size (beyond $180 \mu \mathrm{m}$ ). It was thought some phase separation could have taken place between the coarse particles and the carrier medium during transport studies since the ballotini particles were heavy with a density of $2600 \mathrm{~kg} / \mathrm{m}^{3}$. This was verified by using coarse particles of styrene acrylonitrile (SAN) having a density of $1070 \mathrm{~kg} / \mathrm{m}^{3}$ which was similar to that of the clay suspension used as the medium. The elimination of the density difference between the coarse parsicles and the carrier did not alter the anomalous behaviour. This indicates that there was some structural change occurring within the suspension as the coarse particle size varied. The objective of this study is to investigate this anomalous viscous behaviour and develop a suitable model which will describe the viscosity-coarse particle size relationship.

\section{EXPERIMENTAL}

A bentonite clay suspension ( $3 \mathrm{wt} \%$ clay in water $\simeq 1.2 \mathrm{vol} \%$ ) was used as the carrier medium. It was not a homogeneous fluid but rather a dispersion of fine particles in water. The clay particle size varied between 5 and $100 \mu \mathrm{m}$ with an average size of $55 \mu \mathrm{m}$. The bentonite suspension was stabilised by a procedure ${ }^{5}$ whereby the suspension was sheared for half an hour and rested for a period of 24 hours.

The coarse particles used were coal, ballotini and styrene acrilonitrile (SAN). The particles were divided in the following size ranges : 44-63 $\mu \mathrm{m}, 63-90 \mu \mathrm{m}, 90-125 \mu \mathrm{m}, 125-180 \mu \mathrm{m}, 180-250 \mu \mathrm{m}$ and $250-355 \mu$. The densities of coal, bollotini and SAN were respectively 1400,2600 and $1070 \mathrm{~kg} / \mathrm{m}^{3}$. All rheological measurements were carried out using the cup and bob system of the Contraves Rheomat 115 rheometer. Suspensions of ballotini, coal and SAN were separately prepared by mixing the required amount of the particles into the stabilised bentonite carrier. Concentrations of suspensions prepared varied between 32 and $55 \mathrm{v} / \mathrm{v} \%$. Rheological measurements were carrid out in the shear rate range of 2.3 to $350 \mathrm{~s}^{-1}$. All experiments were performed at $20^{\circ} \pm 1^{\circ} \mathrm{C}$.

\section{RESULTS AND DISCUSSION}

The rheological measurement of bentonite carrier fluid indicated that it was a Bingham type fluid with a yield stress of $6 \mathrm{~Pa}$. The fluid was highly shear thinning with a substantial reduction in apparent viscosity at higher shear $\left(>100 \mathrm{~s}^{-1}\right)$. 
Several viscosity versus particle size relations for coal, ballotini and SAN in bentonite are presented in Figures 1-3. Examination of Figure 1 shows that for 45 vol $\%$ coal in a $3 \mathrm{wt} \%(\approx 1.2 \mathrm{vol}$ $\%)$ bentonite clay suspension, viscosity increases and then decreases with increase in particle size. A similar kind of behaviour is observed for $45 \%$ ballotini in bentonite (Figure 2) although in the later case at low shear rates there are three clear zones i.e. viscosity decrases, then increases and finally decreases with particle size. Similar behaviour has been observed at other concentrations of the coarse particles. The viscosity data of Figure 2 have been plotted as relative viscosity versus

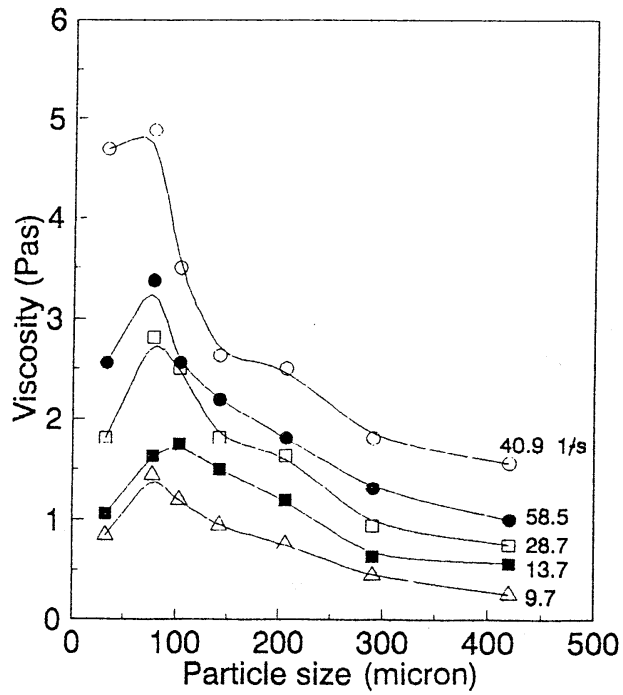

Fig. 1 Viscosity at different shear rate for $45 \%$ coal-bentonite suspension.

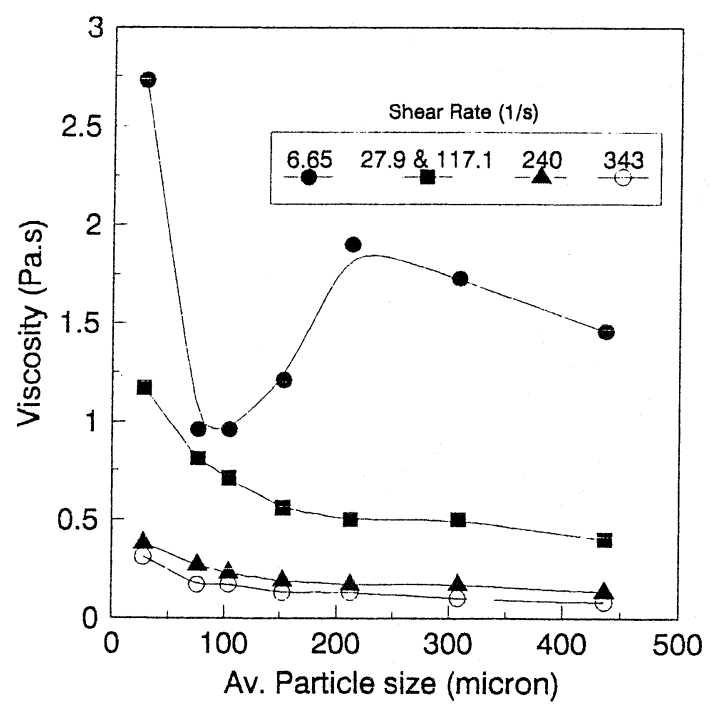

Fig. 3 45\% SAN-bentonite suspension.

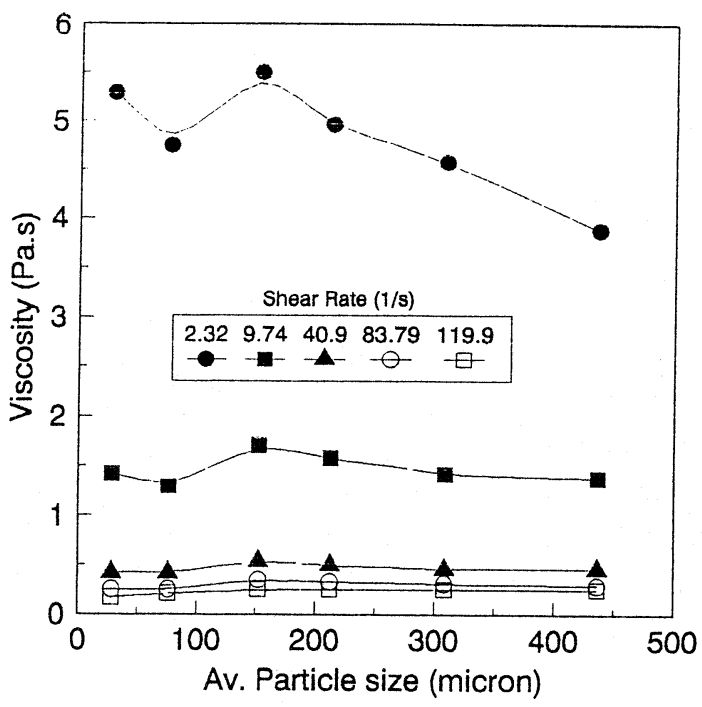

Fig. $245 \%$ ballotini-bentonite suspension. At different shear rates.

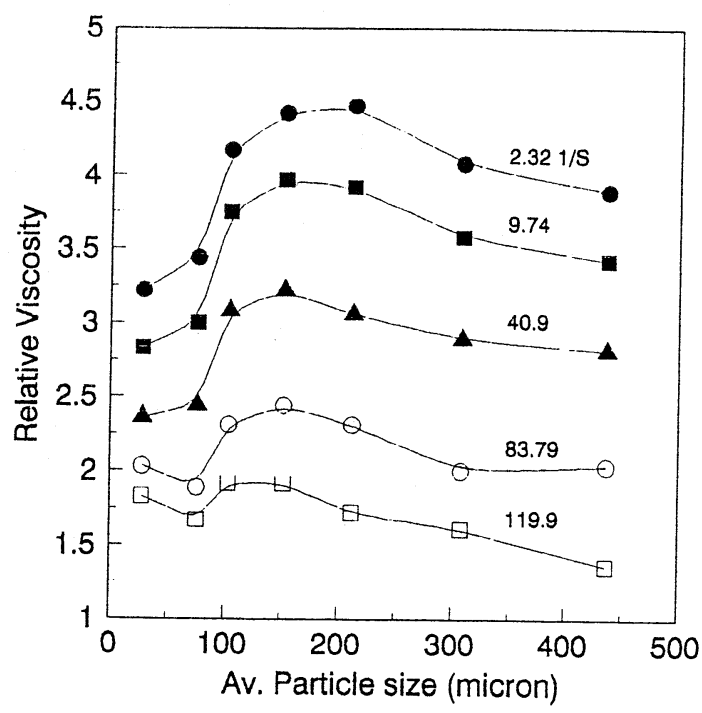

Fig. 4 45\% ballotini-bentonite suspension. 
particle size in Figure 4 ; relative viscosity being defined as the ratio of the coarse suspension viscosity to the bentonite visosity alone at the same shear rate. The date in Figure 4 highlights even more anomalous behaviour with particle size.

From the experimental results in Figures 1-4 the general effect of particle size on viscosity may be represented by dividing the viscosity-particle size diagram into several zones as depicted in Figure 5. Typically these are

i A region of decreasing viscosity with increasing particle size (region A)

ii A region of increasing viscosity with increasing particle size (region $\mathrm{C}$ )

iii A region of decreasing viscosity with increasing particle size (region $\mathrm{E}$ )

iv Two intermediate regions (region $\mathrm{B}$ and $\mathrm{D}$ )

This behaviour is significantly different from the behaviour of any suspension consisting of only fine particles or coarse particles. In those cases viscosity will generally decrease monotonically with increase in particle size ${ }^{4}$.

\section{MODELLING OF SUSPENSION VISCOSITY}

The flow characteristics of clay particles in an aqueous suspension depend on many factors including concdntration, particle size and chemical environment ${ }^{7,8}$. The uneven charge distribution on clay particles produces edge-face and face-face interactions allowing the formation of card house structures and leading to flocculation. The bentonite suspension used in this study is heavily flocculated as evident by a significant decrease in viscosity with the application of increasing shear rate $^{6}$. The addition of coarse particles to bentonite breaks the flocculated structure and the floc size decreases with increase in coarse particle concentration or decrease in particle size. The model is developed based on the following postulates:

i For a given coarse particle size and concentration, the viscosity of bentonite suspension decreases with increase in concentration.

ii For a given concentration of coarse particles bentonite viscosity decreases with decrease in coarse particle size ; as the particle size decreases, the increase in the number of particles is proportional to the cube of the ratio of the initial and final particle size. Since the coarse particles break the floc size, as the number of particles increases, the space between particles decreases resulting in decrease in floc size.

iii At any given concentration and size of the coarse particle, the viscosity of bentonite suspension decreases with shear rate. This thixotropic behaviour is a characteristic of the bentonite suspension.

Based on postulates ( $i$ ) to (iii) the bentonite viscosity may be expressed in the following manner.

$$
\begin{aligned}
& \mu_{\text {bentonite }}=\frac{\mathbf{m}\left(\left|\mathbf{d}_{\text {particle }}-\mathbf{X}\right|\right)^{\mathbf{p}}}{\dot{\gamma}^{\mathrm{q}}} \\
& \text { where } \quad m=A \Phi+B \\
& \boldsymbol{m}=\boldsymbol{C} \Phi+\boldsymbol{D} \\
& \boldsymbol{m}=\boldsymbol{E} \Phi+\boldsymbol{F}
\end{aligned}
$$

$A, B, C, D, E, F$, being constants.

The parameters $\mathrm{m}, \mathrm{p}, \mathbf{q}$, are assumed to be linear functions of $\Phi$, the volumetric concentration 
of the coarse particles. $\dot{\gamma}$ is the applied shear rate and $\mathrm{X}$ is shift factor. An examination of Figures 2 to 4 indicates that this shift factor, corresponding to the first minimum viscosity with respect to the particle size, is approximately $90 \mu \mathrm{m}$. At a particle size less than $90 \mu \mathrm{m}$, particleparticle interaction becomes significant.

The values of the parameters $A$ to $F$ were obtained by fitting the experimental data by a trial and error method and are given in Table 1.

The predictions of this model is compared with the experimental results for SAN and coal in Figures 6 and 7 respectively. Although there is considerable variation between experiments and predictions the model predicts the apparent anomalour behaviour as summarised in Figure 5. This also supports the argument behind this model the viscosity variation of coarse suspension is caused by the modification of the bentonite viscosity. Further study will be required to refine this model.

\section{CONCLUSION}

The viscosity of coarse particle-bentonite suspensions is affected by the size of the coarse particles.

Table 1 Estimated model parameters

$\begin{array}{crr}\text { Parameters } & \text { Coal } & \text { SAN } \\ A & -1.07 & -0.75 \\ B & 0.6 & 0.46 \\ C & -0.6 & -0.85 \\ D & 0.32 & 0.51 \\ E & -0.77 & -1.25 \\ F & 0.85 & 0.98\end{array}$

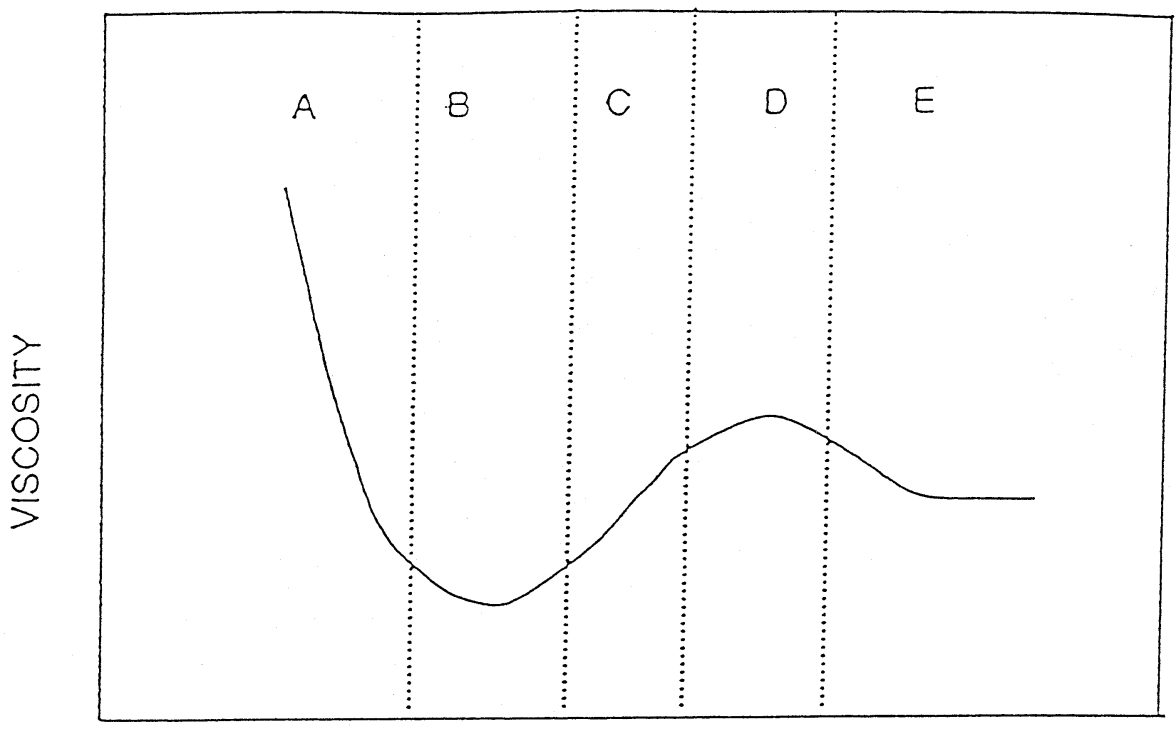

PARTICLE SIZE

Fig. 5 General effect of particle size on viscosity. 


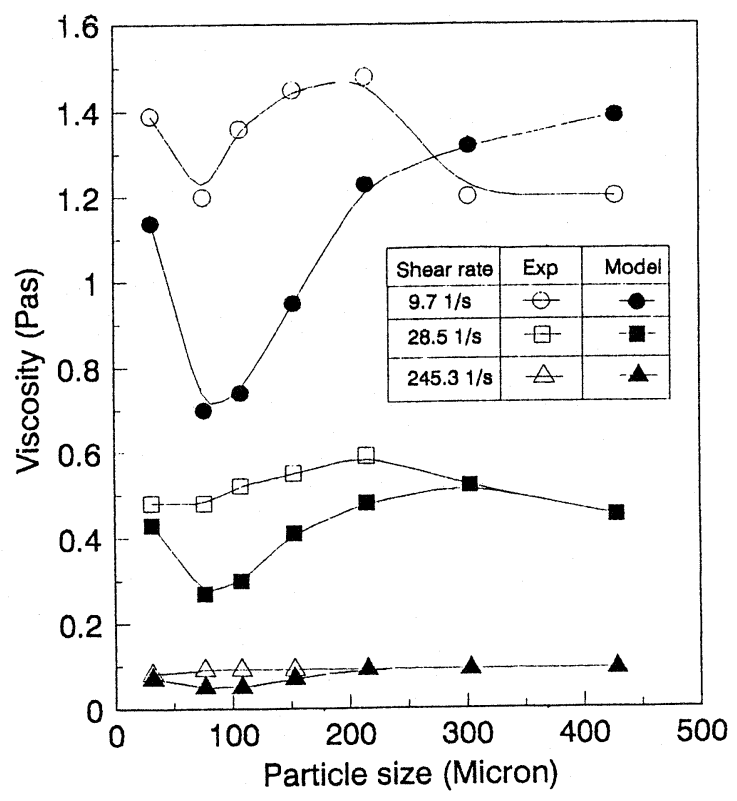

Fig. 6 32\% SAN-bentonite suspension.

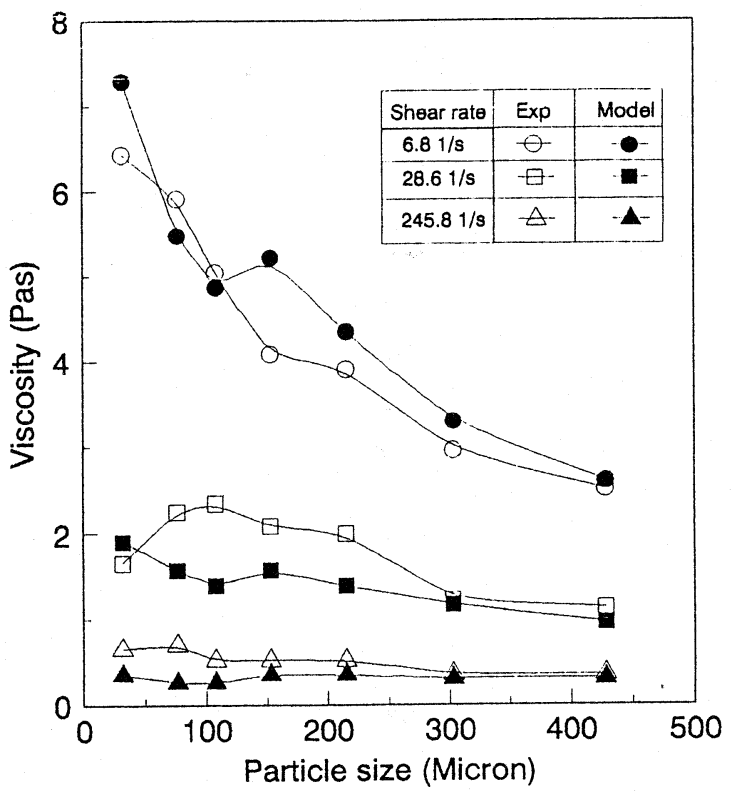

Fig. 7 45\% coal-bentonite suspension.

It is concluded that the viscosity of the carrier fluid, bentonite is affected by the presence of coarse particles. A mathematical model has been developed to predict this behaviour.

\section{ACKNOWLEDGMENT}

The authors wish to thank Australian Research Council for supporting this work.

\section{REFERENCES}

1. Darby, R., Proc. 4th Int. Slurry Transport, 183 (1979)

2. Jinescu, V.V., Int. Chem. Eng. 14, 397 (1974)

3. Clark, B., Trans. Instn. Chem. Engrs., 45, T 251 (1967)

4. Renehan, M.J., Pullum, L. and Bhattacharya, S.N., Xth Int. Congr. Rheo. vol. 2, 207 (1988)

5. Chin, C., O’Dommell, J., Pullum, L. and Bhattacharya, S.N., Chemeca'90, Auckland, NZ, vol. 2, 1148 (1990)

6. Chin, C. O’Dommell, J., Pullum, L. and Bhattacharya, S.N., 16 th Int. Conf. Coal and Slurry Technology, Florida, 459 (1991)

7. Rasekh, H., Rose, K.W. and Worrall, W.E., Brit. Ceramics, Trans. J 86, 132 (1987)

8. Worall, W.E., Clays and Ceramics Raw Materials, Applied Science Publishers (1975) 